\title{
CYTROCONNECT - A CLOUD-BASED IOT-SERVICE AS CONNECTIVITY SOLUTION FOR ELECTROHYDRAULIC SYSTEMS
}

\author{
Martin Laube ${ }^{1 *}$, Andreas Günder ${ }^{1}$, Jan Bierod $^{1}$, Volker Jesberger $^{2}$, Stefan $_{\text {Rauch }}^{2}$ \\ ${ }^{1}$ Bosch Rexroth AG, Zum Eisengiesser 1, 97816 Lohr a. Main \\ ${ }^{2}$ grow platform GmbH, Postfach 3002 40, 70442 Stuttgart \\ * Corresponding author: Tel.: +49 9352 183619; E-mail address: martin.laube@boschrexroth.de
}

\begin{abstract}
Conventional electrohydraulic solutions integrate easily into modern machine concepts by utilizing field bus technology. Nevertheless, most use cases are limited to machine automation concepts. Integration into higher-level data and IoT systems is the key for positioning of electrohydraulic solutions within the factory of the future. CytroConnect is a new approach for the integration of electrohydraulic systems into IoT environments and the corresponding market offerings. Bosch Rexroth decided not only to integrate IoT-ready features like pre-installed sensor packages but also a modular automation concept providing decentralized intelligence with an open multi-ethernet interface. An edge-to-cloud connectivity stack operated by Bosch turns the target into a Connected Product. The convergence of physical and digital product can be realized. Based on that the digital service CytroConnect solves concrete holistic use cases like visualization and condition monitoring by offering a web-based dashboard of all relevant sensor data that is accessible everywhere. Modular paid add-ons offered as risk-free monthly subscriptions address further smart maintenance and prediction use cases.
\end{abstract} Keywords: CytroBox, IoT, Connectivity, Industry 4.0, electrohydraulic systems

\section{STATE-OF-THE-ART}

Pulled by the increasing complexity and variances in products and pushed by the progressive capabilities in communication technology the internet and automation systems are getting more and more connected to reach a new level of efficiency.

The traditional way of industrial communication is described in the automation pyramid (Figure). The exchange of data is mainly between the adjacent levels. Due to the diverse manufacturers related standards it is taking a lot of effort to realize level crossing communication. With standards like OPC-UA and Ethernet TNS, it is possible to exchange data between different systems and automation levels efficient and in real time, which is important for M2M communication in an industry 4.0 environment.

The communication of traditional electrohydraulic drive systems is mainly field bus based and only connecting the different components of the drive system to the machine control. It is a clear focus on realizing the machine functionalities and required performance. The data is not leaving the machine border and only basic and no scalable condition-monitoring concepts are integrated.

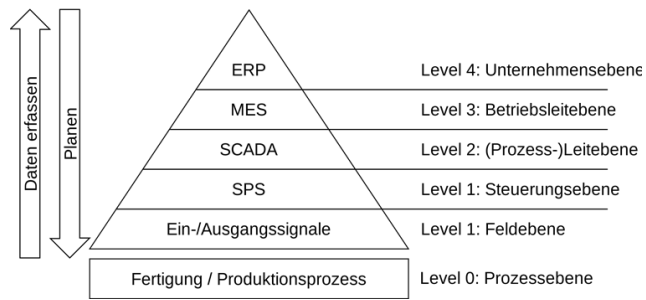

Figure 1: Automation Pyramid [1]

\section{MOTIVATION FOR NEW CONNECTIVITY SOLUTIONS}

Physical products and digital services merge into hybrid solutions. Physical products become carriers of digital services, also known as Smart Components, for which services and business models are offered on a "Smart Service Platform" in the form of "Smart Services".

These "Smart Services" for example, can 
enable fast machine service or other business models such as pay-per-use for machines and components. It should be possible to use engineering data and live data from the machines and components to develop the "Smart Services". In order to be able to add quickly new functionalities and services to the systems, it is essential to collect and prepare data in a cloud and to use a suitable device management. This platform approach enables the scalable further implementation of technical features and the integration of new use cases. By means of a standardized building block of physical products and systems, digital services can be transferred to

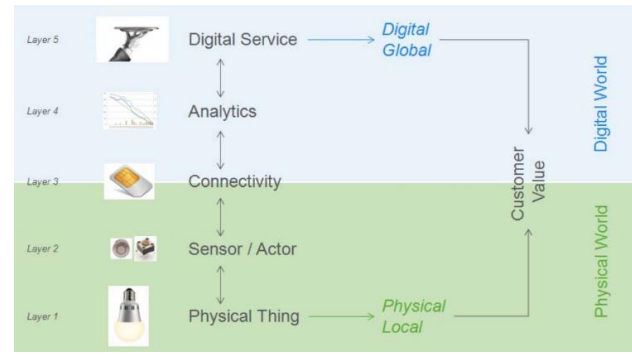

different hardware products with little effort.

Figure 2: Value-creation Layers in an Internet of Things Application [2]

As shown in the figure below (Figure 3), physical products with their digital services are thus establishing themselves as drivers for new maintenance and service concepts and for new business models. The targeted collection and analysis of data is becoming increasingly important and is a key capability for product and business model design. Examples are provided here by the business model patterns subscription, flat rate, freemium, pay per use and performancebased contracting.

As an additional benefit of collected data, the conditions of use and business models also change in physical services (Figure 4). For example, in the event of a fault, service technicians can use the data to carry out a faster diagnosis and data-supported business models can also be offered here.

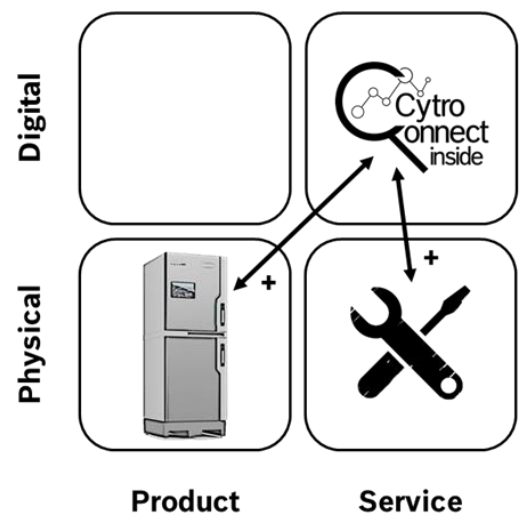

Figure 4: Digital Add-ons enlarge customer benefits

To leverage the potential in IoT technologies, it is crucial that the number of systems sold is decoupled from the size of the team responsible for developing and operating the solution. It is therefore crucial to consider the scaling aspect in

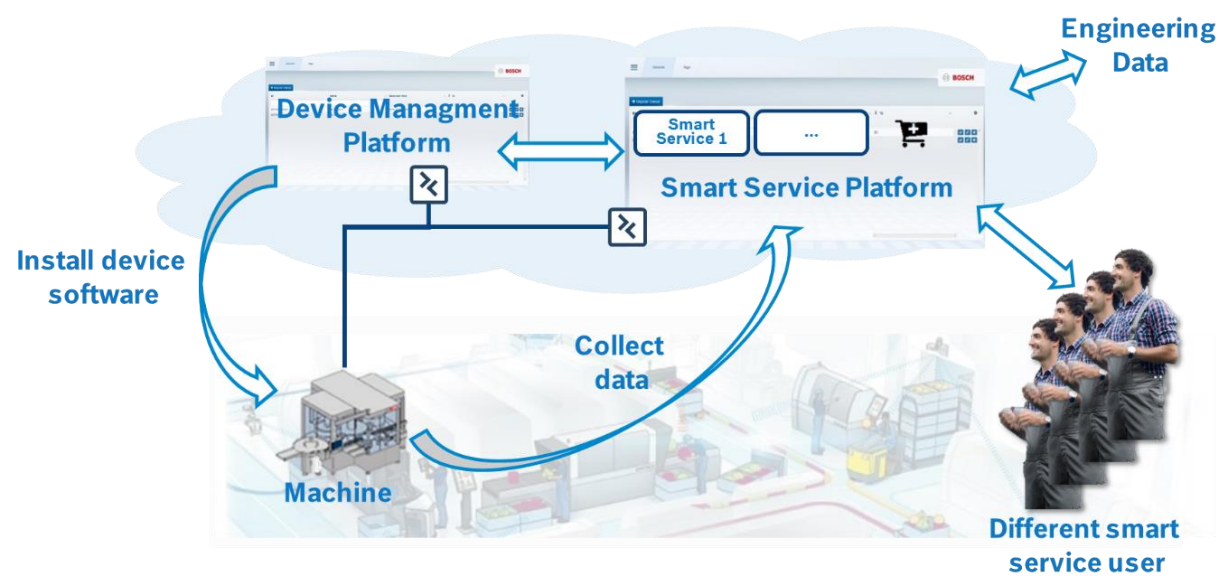

Figure 3: Concept Smart Service [2] 
the solution from the very beginning in the design. The project planning and commissioning processes must also be taken into account. The goal must be to automate them completely. Another aspect that also contributes to the topic of "Non-Human-Scaling" is the topic of Plug and Play. If products do not generate any commissioning effort and the customer does not have to make any adjustments to the configuration for commissioning (because this all happens automatically), the service and support effort (both scaled by humans) can also be minimized on the manufacturer side.

\section{CYTROCONNECT AS A NEW APPROACH}

Based on the requirements driven by the technical concepts for convergence of physical and digital products Bosch Rexroth has developed and implemented a new solution for the integration of electrohydraulic systems into IoT environments and the corresponding market offerings which is described in the following:

\subsection{Hardware Concept}

The concept is physically divided into the hardware integrated in the target product (sensor technology, data acquisition unit) and corresponding edge devices (communication module) Figure 5.

A PLC integrated in the target product is the main control system. Sensors in the system exposing their data via IO-Link to an IO-LinkMaster, that communicates with the PLC via Fieldbus. For decoupling- and IT-security- purposes, a dedicated industrial PC (IPC) is used as the edge device for the IoT services. This also brings the advantage, that a different (Linux based) software platform can be used to realize data streaming and IT security requirements.

\section{Browser based UI}

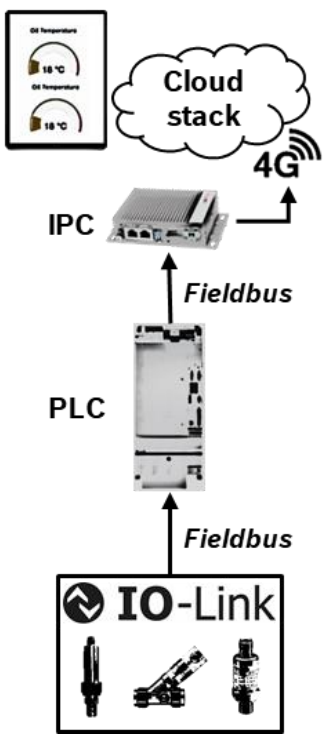

Figure 5: Hardware Topology (Product, Edge Device, Cloud, User Interface) [3]

\subsection{Software Topology}

Each target product is equipped with a dedicated Industrial-PC for exposing data to IoT-Services. This device is capturing sensor- and condition data from the control system (PLC) of the target product and pushes it via 4G-Link to a cloud

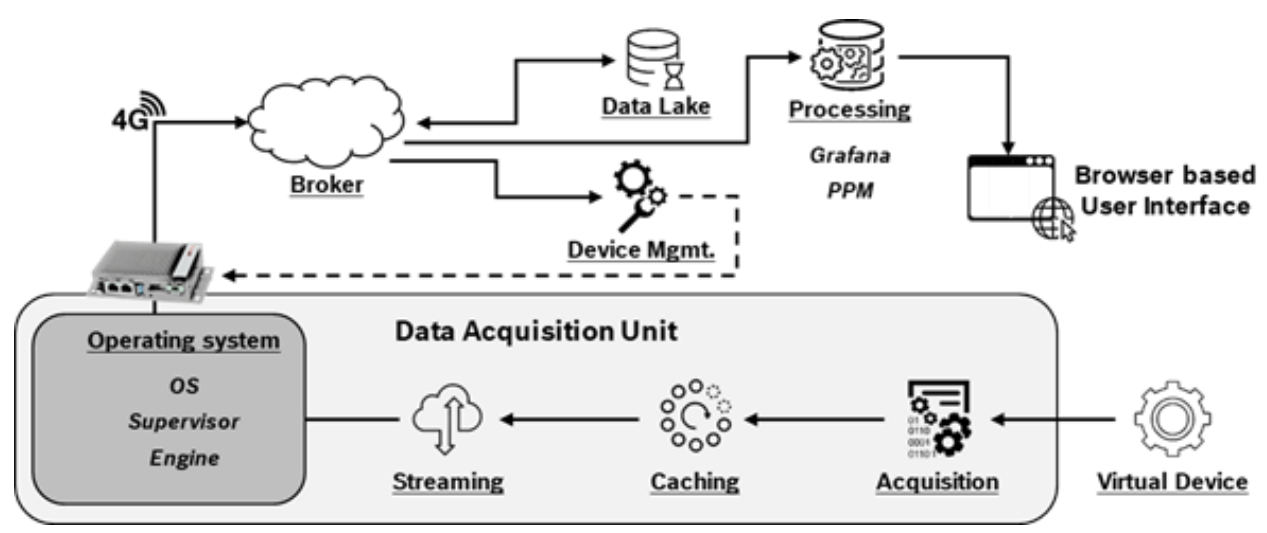

Figure 6:

Software topology CytroConnect 
endpoint. The data of all devices in the deployed fleet is streamed to a broker that is used as the central data hub. To make the data available in the customer facing systems, filtering rules and data transformations are applied on the data streams Figure 6.

Beside the customer facing solutions (a customizable Grafana Dashboard and the Bosch Nexeed Production Performance Manager PPM), there are some additional systems, that are required for running such a platform. A Monitoring solution that allows to monitor the health of the infrastructure, a Device Management solution, which enables software updates and management of the edge devices and a Data Lake that is storing raw data for intended analytics use cases. The dashboard, showing the machine data is a web application, running in the cloud. That enables access to machine data by any device, every time and everywhere.

\subsection{Digital Service as a business model}

The integrated IoT service Bosch Rexroth CytroConnect is intended to ensure higher availability and to avoid unplanned downtimes. With this IoT service, operators can monitor operating state and plan maintenance in a costefficient manner. As a result, fluid technology has taken a further step towards the factory of the future.

With the CytroConnect digital service, operators have all information about their product all time available. Whether it is the visualization of the component and operation state or chargeable IoT services. This services - based on big data analytics - support the maintenance work during troubleshooting and provide warnings of upcoming events before they happen. In addition to the condition monitoring of the target product, maintenance personnel and maintenance managers can add extra solutions for various applications as add-ons. These payper-use payment models include additional IoT analytics tools and can be subscribed to on a monthly basis.

For optimizing maintenance processes, the CytroConnect Maintain module offers access to historical sensor data as well as instant messages if maintenance is required. Pre-defined rules based on long time application experience allows customers to improve their maintenance strategy on an ongoing basis. With that combination of scalable software, Bosch IoT solutions and Bosch Rexroth domain specialists a high benefit can be offered to the customers.

One example of the used Bosch IoT solutions is the Nexeed Production Performance Manager (PPM). It is a software solution for systematic production optimization and allows easy processing of acquired data. With that tool, the different components and data can be set into correlation. Single sensor or data points like pressure, oil temperature or level for themselves don't allow conclusions about the system. However, in correlation the data sources can be used to perform a condition diagnosis of the system.

Beyond this, the CytroConnect Predict service offers the possibility of using machine learning. Big data analytics and self-learning algorithms calculate the actual status and expected remaining lifetime of relevant components. Based on this the operator gets informed and can plan the downtime. Furthermore, this information allows changing the component in advance to prevent further damage on the complete system and ensures maximum availability.

\section{MATURITY AND VALIDATION}

To evaluate the entire concept a first target product was defined - the new standardized hydraulic power unit CytroBox. Looking on the hardware topology in Figure 7 it shows why this product is perfectly suitable for a digital service. By means of the newest version of speed variable servo technology in combination with IO-Link sensors, it is possible to collect many different data points of all components. Based on this architecture the entire soft- and hardware stack was built, always focused on an open and scalable design.

Already in the CytroBox development process, CytroConnect could be used in order to detect unknown behavior of the sample units during testing and for troubleshooting. For example, the detection of a damaged pressure sensor allowed the development team to order and replace it, even before there was a failure of the system. Further issues during testing and validation of the units in customer prototype machines like system leakage caused by a defect cylinder could be detected and evaluated by CytroConnect. Fixing of these incidents could happen without having the development team on- 


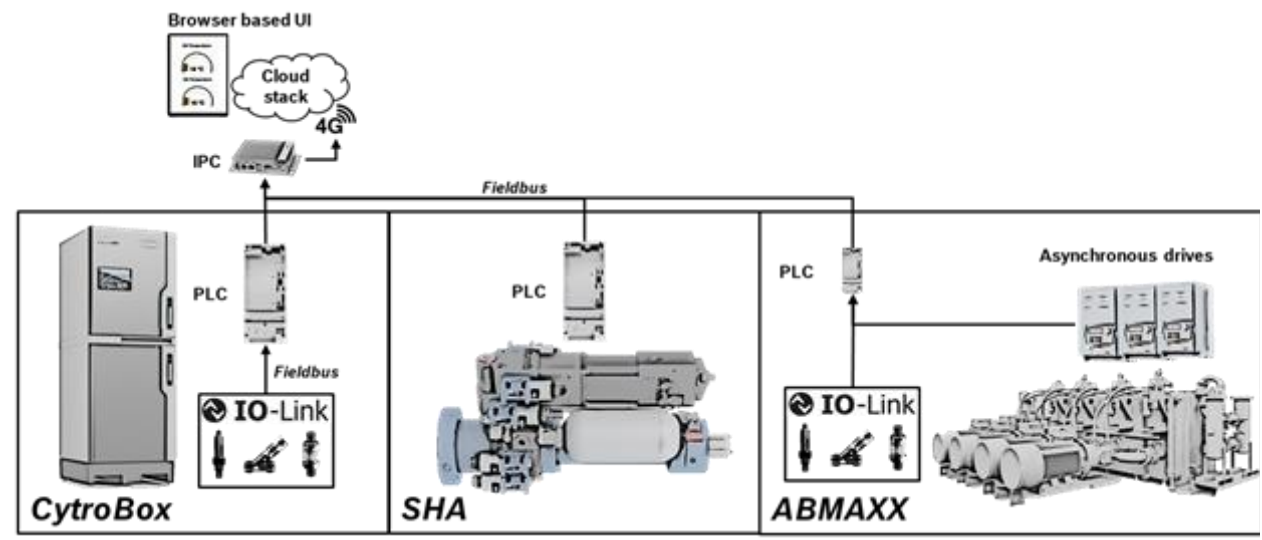

Figure 7: Hardware Architecture CytroConnect portfolio extension

site, just by a phone call in most cases.

Depending on this experience, the actual target for CytroConnect is to extend the supported product portfolio. Due to the open design and scalability it's easy to implement into different electrohydraulic system solutions, like the Servo Hydraulic Actuators (SHA) or Modular Large HPU (e.g. ABMAXX). Figure 7 shows the needed hardware architecture for different target products. PLC and edge device integrated in the target products are kept identical throughout all possible use cases. To implement the different products on the cloud no main software changes are required. Only the dashboard for visualization and the product specific rules need to be configured and adjusted.

\section{SUMMARY AND BUSINESS OUTLOOK}

Driving digitalization into industrial hydraulics is clear focus topic in order to offer beneficial solutions in the environment of Industry 4.0 to the market. Besides providing products that are able to be digitally connected to superior control systems the combination with enabling these products for digital services and the operation of corresponding digital structures offers the possibility of additional data driven business models. With CytroConnect Bosch Rexroth offers a digital service platform, which allows electrohydraulic systems solutions to be integrated in the environment of the Factory of the Future. Online monitoring of operation conditions, remote diagnosis and maintenance as well as predictive services based on big data analytics become possible. The related data driven business models like subscription, flat rate, freemium, pay per use and performancebased contracting allow advanced operation and maintenance concepts for OEMs and End-Users. The key success factor in the CytroConnect concept is the simple scalability and extension of the Hardware and Software concept on various target products, which allows consistent appearance for the user throughout the complete product portfolio. Having the well-known competition of drive technologies in mind, concepts like CytroConnect bring industrial hydraulics significantly forward and allow it to become part of the Factory of the Future.

\section{REFERENCES}

[1]https://de.wikipedia.org/wiki/Automatisierungs pyramide

[2] Bosch Internet of Things \& Services Lab, St. Gallen

[3] io-link.com 